Portland State University

PDXScholar

Electrical and Computer Engineering Faculty

Publications and Presentations

$11-2002$

\title{
Source Localization in a Time-Varying Ocean Waveguide
}

Cristiano Soares

Universidade do Algarve

Martin Siderius

Portland State University, siderius@pdx.edu

Sérgio M. Jesus

Universidade do Algarve

Follow this and additional works at: https://pdxscholar.library.pdx.edu/ece_fac

Part of the Electrical and Computer Engineering Commons

Let us know how access to this document benefits you.

\section{Citation Details}

Soares, C., Siderius, M., \& Jesus, S. (2002). Source localization in a time-varying ocean waveguide. The Journal of The Acoustical Society of America, 112(5 Pt 1), 1879-1889.

This Article is brought to you for free and open access. It has been accepted for inclusion in Electrical and Computer Engineering Faculty Publications and Presentations by an authorized administrator of PDXScholar. Please contact us if we can make this document more accessible: pdxscholar@pdx.edu. 


\title{
Source localization in a time-varying ocean waveguide
}

\author{
Cristiano Soares ${ }^{\text {a) }}$ \\ Faculdade de Ciências e Tecnologia, Universidade do Algarve, Campus de Gambelas, 8000-Faro, Portugal \\ Martin Siderius ${ }^{\text {b) }}$ \\ SACLANT Undersea Research Centre, Viale S. Bartolomeo 400, 19138 La Spiezia, Italy
}

Sérgio M. Jesus ${ }^{\text {() }}$

Faculdade de Ciências e Tecnologia, Universidade do Algarve, Campus de Gambelas, 8000-Faro, Portugal

(Received 4 September 2001; revised 15 July 2002; accepted 29 July 2002)

\begin{abstract}
One of the most stringent impairments in matched-field processing is the impact of missing or erroneous environmental information on the final source location estimate. This problem is known in the literature as model mismatch and is strongly frequency dependent. Another unavoidable factor that contributes to model mismatch is the natural time and spatial variability of the ocean waveguide. As a consequence, most of the experimental results obtained to date focus on short source-receiver ranges (usually $<5 \mathrm{~km}$ ), stationary sources, reduced time windows and frequencies generally below $600 \mathrm{~Hz}$. This paper shows that MFP source localization can be made robust to time-space environmental mismatch if the parameters responsible for the mismatch are clearly identified, properly modeled and (time-)adaptively estimated by a focalization procedure prior to MFP source localization. The data acquired during the ADVENT'99 sea trial at 2, 5, and $10 \mathrm{~km}$ source-receiver ranges and in two frequency bands, below and above $600 \mathrm{~Hz}$, provided an excellent opportunity to test the proposed techniques. The results indicate that an adequate parametrization of the waveguide is effective up to $10 \mathrm{~km}$ range in both frequency bands achieving a precise localization during the whole recording of the $5 \mathrm{~km}$ track, and most of the $10 \mathrm{~km}$ track. It is shown that the increasing MFP dependence on erroneous environmental information in the higher frequency and at longer ranges can only be accounted for by including a time dependent modeling of the water column sound speed profile. (c) 2002 Acoustical Society of America.
\end{abstract}

[DOI: $10.1121 / 1.1508786$ ]

PACS numbers: 43.30.Wi, 43.60.Pt, 43.30.Pc [DLB]

\section{INTRODUCTION}

Matched-field processing (MFP) is an inversion method that allows a source to be located from receptions on an array and has mostly been applied to sound sources in the ocean ${ }^{1,2}$ (see also Ref. 3 and references therein). The MFP technique compares the received field with replica fields generated for all possible source locations using an acoustic propagation model. Localization results degrade when inaccurate or insufficient data is used as input to the propagation model. This is often called the model mismatch problem and can occur when environmental information, such as the ocean sound speed, is not known in sufficient detail. Mismatch also occurs when there is uncertainty in the measurement geometry, such as receiver array position. ${ }^{4,5}$ In classical MFP the environment and measurement geometry are assumed known and the inversion search space includes only parameters relating to the source location.

To mitigate model mismatch, a class of matched-field processors, including the so-called uncertain processors (OFUP $)^{6}$ and the focalization processor, ${ }^{7}$ emerged in the past decade. These processors include both environmental and

\footnotetext{
${ }^{\text {a)} E l e c t r o n i c ~ m a i l: ~ c s o a r e s @ u a l g . p t ~}$

b)Electronic mail: thomas.martin.siderius@saic.com; Current address: Science Applications International Corporation, 10260 Campus Point Dr., San Diego, CA 92121.

${ }^{c)}$ Electronic mail: sjesus@ualg.pt
}

geometric parameters in the search space, hence reducing potential model mismatch problems. [Throughout this paper geometric parameters are those related to source and receiver positions, as well as propagation channel dimensions (e.g., water depth). Environmental parameters are those that characterize acoustic properties of the propagation channel (e.g., seabed sound speed).] It has been shown that these processors can successfully locate acoustic sources in the ocean even if the environmental knowledge is limited. ${ }^{8-10}$

Focalization and OFUP should perform well if the most important environmental and geometric parameters are included in the search space. This becomes more difficult as source frequency increases and therefore the relevant time and length scales needed to describe the environment decrease. This is one reason why most experimental studies on both classical MFP and focalization have been applied to data at frequencies below $500 \mathrm{~Hz}$. However, sound sources in the ocean often occur at frequencies above $500 \mathrm{~Hz}$. The sources may be natural (e.g., from marine life) or man-made (e.g., engines or sonar sound projectors). The main issue being addressed in this paper is to experimentally test whether MFP can be applied in a shallow water scenario at frequencies up to $1500 \mathrm{~Hz}$. It will be shown that at these higher frequencies, the variability of the water column sound speed plays an important role that needs to be included in the processing.

The data considered here was collected in May, 1999 


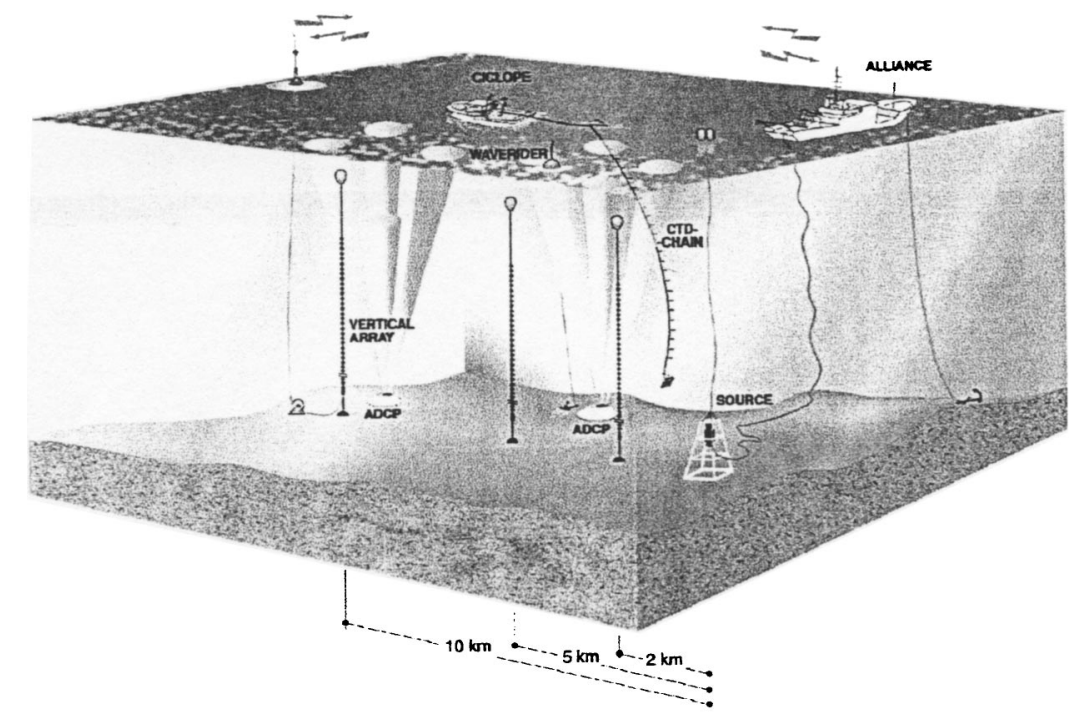

FIG. 1. ADVENT'99 sea trial setup with three vertical line array at positions 2,5 , and $10 \mathrm{~km}$ from a bottom mounted acoustic source. R/V Alliance is transmitting acoustic signals and collecting vertical line array data through an RF link. ITNS Ciclope is towing a CTD chain along the acoustic transmission track.

during the ADVENT'99 sea trial. One of the goals of this experiment was to test the performance of field inversion methods in shallow water under controlled conditions. The signals emitted were multitones (MT) and linear frequency modulated (LFM) sweeps in the bands 200-700 Hz and 800-1600 Hz. A vertical array was deployed on three separate days at ranges of 2,5 , and $10 \mathrm{~km}$ from the source.

In Ref. 11, Siderius et al. performed MFP inversion (focalization) on the ADVENT'99 low frequency data. The data from 2 and $10 \mathrm{~km}$ ranges were inverted for both the source location and seabed properties. Time- and range-independent water column sound speed profiles were used for each of the two tracks considered. Their results indicate that for the $2 \mathrm{~km}$ track a consistent high correlation between measured and modeled broadband data can be obtained and this resulted in reliable estimates for the source location and seabed properties. Inversion of data taken at $10 \mathrm{~km}$ showed increased variability in estimates for both source position and seabed properties. The conclusion is that the environmental variability can destroy coherent processing and propagation prediction of acoustic data leading to erroneous estimates for source location and seabed properties. In the case of the ADVENT'99 data this was true for the low frequency data at $10 \mathrm{~km}$ source receiver separation.

This paper reports successful source localization results using the ADVENT'99 data taken at ranges of 2, 5, and 10 $\mathrm{km}$ and up to frequencies of $1500 \mathrm{~Hz}$. This is achieved through a focalization process that accounts for the time and space variability of the environment (including ocean sound speed structure). The focalization search space is directed using a genetic algorithm (GA). ${ }^{12}$ As a further demonstration of the utility and robustness of the method, localization was tested using data from short arrays (subapertures from the ADVENT'99 vertical array). Successful application of MFP to short arrays with few sensors is important for practical applications since long, vertical arrays may not always be available. Results will be presented in Sec. III showing successful source localization in the $800-1600 \mathrm{~Hz}$ band at $5 \mathrm{~km}$ range using four sensors with a total vertical aperture of just $8 \mathrm{~m}$. Vertical apertures of this size may be possible even from the droop of a horizontally towed array.

\section{THE ADVENT'99 EXPERIMENT}

During the first three days of May of 1999 the ADVENT'99 experiments were conducted by the SACLANT Undersea Research Center and TNO-FEL on the Adventure Bank off the southwest coast of Sicily (Italy). Figure 1 shows a drawing of the experimental setup. The bathymetry in the area has slight variations but the average water depth is $80 \mathrm{~m}$. The acoustic sources were located at 76 $\mathrm{m}$ depth, mounted on steel framed tower that was sitting on the seabed. The signals were received on a $62 \mathrm{~m}-32$ hydrophone vertical array that was deployed at ranges of 2, 5, and $10 \mathrm{~km}$. Only data from 31 hydrophones are considered in this paper (element 25 is missing). Broadband LFM and MT signals were transmitted using two sound projectors, one for lower frequencies $(200-700 \mathrm{~Hz})$, and another for higher frequencies $(800-1600 \mathrm{~Hz})$. The transmission time was around 5 hours for the 2 and $5 \mathrm{~km}$ tracks, and 18 hours for the $10 \mathrm{~km}$ track. The data used here has an estimated signal-to-noise ratio greater than $10 \mathrm{~dB}$.

For sound speed measurements a 49-element conductivity-temperature-depth (CTD) chain was towed by ITNS Ciclope. The CTD chain spanned around $80 \%$ of the water column reaching a maximum depth of $67 \mathrm{~m}$, and was continuously towed between the acoustic source and the vertical array (10 km track). The data was sampled every $2 \mathrm{~s}$ which corresponds to a vertical sound speed profile measurement approximately every $4 \mathrm{~m}$ in range. See Refs. 13 and 14 for a more detailed description of the experiment.

\section{PARAMETER ESTIMATION USING GA AND SOURCE LOCALIZATION}

The focalization procedure proposed by Collins et al. ${ }^{7}$ was adopted in this study to account for time-varying environments. This was accomplished using the following twostep algorithm:

(1) GA estimation of geometric and environmental param- 


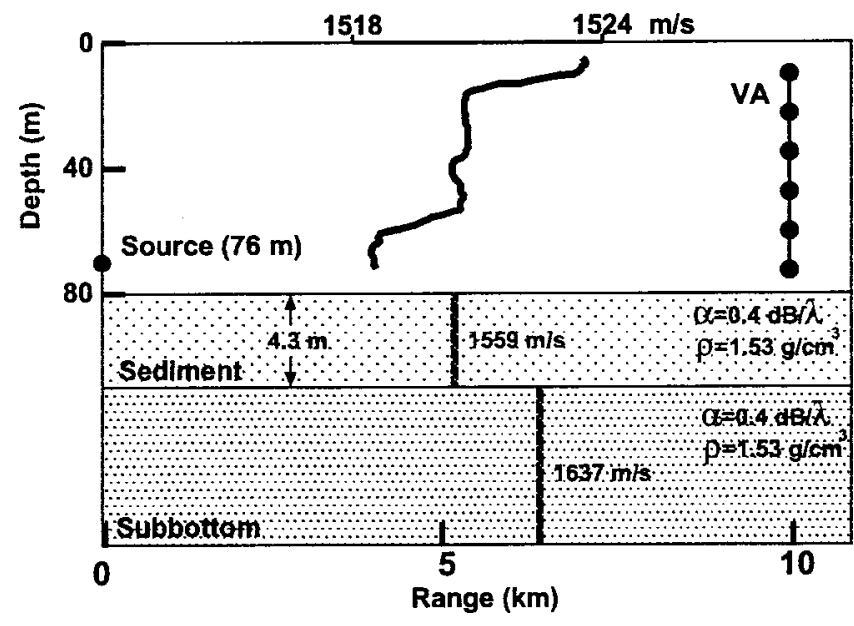

FIG. 2. Baseline model for the ADVENT'99 experiment. All parameters are range independent. The model assumes the same density and attenuation for sediment and sub-bottom.

eters: in order to determine (focus) the array position and environment to be used in the source localization-step (2).

(2) Exhaustive range-depth source localization with parameters obtained in step (1).

The matched-field localization problem usually does not include step (1) since the geometric (e.g., array position) environment (e.g., sound speed profile) and bathymetry are assumed known. There is, however, always some uncertainty in these parameters and step (1) is included here to accurately determine the optimal array position, effective bathymetry, and sound speed before application of the step(2) localization.

One of the difficulties associated with any MFP study is the choice of the environmental model used to represent the real environment where the acoustic signal propagates. Here, we define a baseline model that contains a mathematical description of the real environment and constrains the attainable set of solutions.

\section{A. The baseline model}

The baseline model consists of an ocean water column overlying a sediment layer and a bottom half-space, assumed to be range independent, as shown in Fig. 2. For step (1), the forward model parameters were divided into two subsets: geometric parameters and water column parameters. The geometric parameters include source range, source depth, receiver depth, array tilt, and water depth. The parametrization of the water column will be explained later. The baseline sediment and bottom properties used for the experimental site were those estimated by Siderius et al. ${ }^{11}$ using the low frequency data set. Figure 2 shows an example of a sound speed profile measured close to the vertical array at $06: 38$ on May 2. This profile is typical of those measured during the experiment showing a double thermocline at 10 and $55 \mathrm{~m}$ depth with isovelocity layers in between.

\section{B. The objective function}

The focalization of the environment and geometry [step (1)] was posed as an optimization problem, that is, to find a vector of parameters $\underline{\theta}$ that maximizes an objective function. The objective function used here is the conventional frequency incoherent broadband processor, also called the Bartlett processor, and is defined as

$$
P(\underline{\theta})=\frac{1}{N} \sum_{n=1}^{N} \underline{p}^{H}\left(\underline{\theta}, \omega_{n}\right) \hat{\mathbf{C}}_{X X}\left(\omega_{n}\right) \underline{p}\left(\underline{\theta}, \omega_{n}\right) .
$$

The factor $\hat{\mathbf{C}}_{X X}\left(\omega_{n}\right)$ is the sample cross-spectral matrix obtained from the observed acoustic field at frequency $\omega_{n}, N$ is the number of frequency bins, and $p$ are the replica vectors to be matched with the data. All factors in (1) have norm equal 1 , hence the maximum attainable value of $P(\underline{\theta})$ is 1 .

The cross-spectral matrices were computed from the time series received on the 31 hydrophones using the lower frequency tones (200 to $700 \mathrm{~Hz}$ with $100 \mathrm{~Hz}$ spacing) and higher frequency tones $(800,900,1000,1200,1400,1500$ $\mathrm{Hz}$ ). Each ping of $10 \mathrm{~s}$ was divided into $0.5 \mathrm{~s}$ nonoverlapping segments, where the first and last segments were discarded, giving a total of 18 segments. Then, the 18 data segments were Fourier transformed, the bins corresponding to the multitone frequencies extracted, and the sample cross-spectral matrices computed. This procedure was repeated every 28 minutes for the $2 \mathrm{~km}$ track and at every 32 minutes for the 5 and $10 \mathrm{~km}$ tracks, for a total of 12 estimates for each track. Note that, for convenience and perceptibility, the ambiguity surfaces shown throughout this paper are only the odd numbered surfaces out of the 12 estimates.

\section{Model parameter estimation and source localization}

To cope with the time variability of the acoustic field the source-receiver geometry and the environmental parameters were optimized using genetic algorithm (GA) search. The GA settings were adjusted as follows: the number of iterations was set to 40 with three independent populations of 100 individuals; crossover and mutation probabilities were set to 0.9 and 0.011 , respectively. These GA settings were slightly re-adjusted as additional environmental parameters were included to the search space throughout this study.

The GA optimization was carried out using a varying number of environmental parameters depending on the track. Throughout this paper it will be explained which environmental and geometric parameters were included in each inversion, but only source localization results are reported. There is no intention of validating the environmental parameters obtained throughout the various propagation tracks. The focalization step can produce a set of optimized parameters that are not necessarily correct even with a match of the replica field with the array received field. In this case a so-called equivalent model is obtained.

Table I shows the geometric parameters of the forward model and their respective search bounds for each range track. Note that source and receiving array depth are coupled to water depth, and therefore they are referenced to the depth of the bottom and referred to as source and array height.

For each source range, GA optimization was first carried out for the lower frequency MT, where the sensitivity to model mismatch is expected to be lower. Then source local- 
TABLE I. Search bounds for the GA optimization of the geometric parameters; source and height of the deepest receiver on the array are measured from the water-sediment interface and are therefore coupled with the water depth search parameter. Water depth was only searched for the $10 \mathrm{~km}$ track and held fixed to $80 \mathrm{~m}$ for the 2 and $5 \mathrm{~km}$ tracks.

\begin{tabular}{|c|c|c|c|c|c|c|}
\hline \multirow[b]{2}{*}{ Parameter } & \multicolumn{2}{|c|}{$2 \mathrm{~km}$} & \multicolumn{2}{|c|}{$5 \mathrm{~km}$} & \multicolumn{2}{|c|}{$10 \mathrm{~km}$} \\
\hline & $\min$ & $\max$ & $\min$ & $\max$ & $\min$ & $\max$ \\
\hline Source range $(\mathrm{km})$ & 1.8 & 2.6 & 4.7 & 5.8 & 10.0 & 11.0 \\
\hline Source height (m) & 1 & 10 & 1 & 10 & 1 & 10 \\
\hline Array height (m) & 1 & 15 & 1 & 15 & 1 & 15 \\
\hline Array tilt (rad) & -0.025 & 0.025 & -0.025 & 0.025 & -0.025 & 0.025 \\
\hline Water depth (m) & 80 & 80 & 80 & 80 & 78 & 82 \\
\hline
\end{tabular}

ization ambiguity surfaces were generated for both frequency bands using replicas computed using the parameters estimated in the GA optimization. All replicas were computed using the SACLANTCEN normal mode propagation code C-SNAP. ${ }^{15}$

\section{The $2 \mathrm{~km}$ track}

Since model mismatch is less problematic at shorter ranges, the optimization [step (1)] for the $2 \mathrm{~km}$ track data set was performed directly on the higher frequency MT. The step-(1) parameter bounds for optimizing the array position are shown in Table I. The water column sound speed profile was linearly extrapolated down to the bottom using the two deepest sound speed values. No optimization was performed for the water depth since no significant water depth changes were expected in the relatively short range of $2 \mathrm{~km}$.

The step-(2) range-depth ambiguity surfaces were computed for source ranges varying from 1 to $3 \mathrm{~km}$, and source depth between 10 and $80 \mathrm{~m}$ using the baseline environmental parameters and the previously GA estimated geometry. Figure 3 shows only the odd numbered ambiguity surfaces, and Table II summarizes the results obtained through time in terms of the mean and standard deviation. The time elapsed between surfaces is 28 minutes except between surfaces 2 and 3 (where transmissions were interrupted). All surfaces show a relatively stable ambiguity pattern with a clear peak standing out from the background at the correct source position. The standard deviation is low for the source parameters and the Bartlett power has mean of 0.57 (Table II). This relatively low Bartlett power can be explained by the large frequency band being used: in order to get the maximum average match, the model has to find a parameter set that yields the best overall matched-field response, but not the best single-frequency matched-field response. If the optimization was carried out separately for each frequency, the optimum parameter set would be different and a higher matched-field response would therefore be attained for each frequency. In Fig. 4 a comparison is shown between the replicas and the received field for the tones in the best and worst match case. The best match is generally obtained at $900 \mathrm{~Hz}$ (0.77) and the worst match is obtained for $1500 \mathrm{~Hz}(0.56)$.

A full optimization including all seafloor parameters has shown little dependence of the acoustic field in this frequency band. Moreover, fluctuations in the propagation
$(1, \mathrm{~B}=0.70)$

$(3, \mathrm{~B}=0.50)$

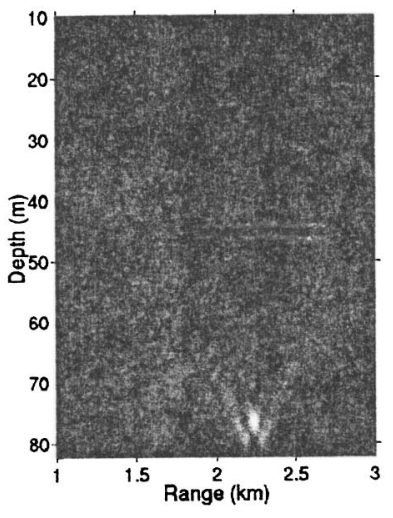

$(5, \mathrm{~B}=0.58)$

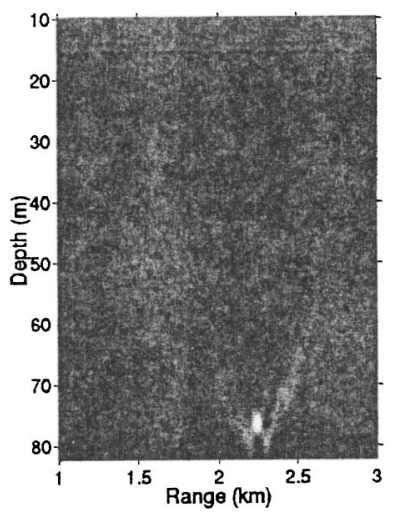

$(9, \mathrm{~B}=0.63)$
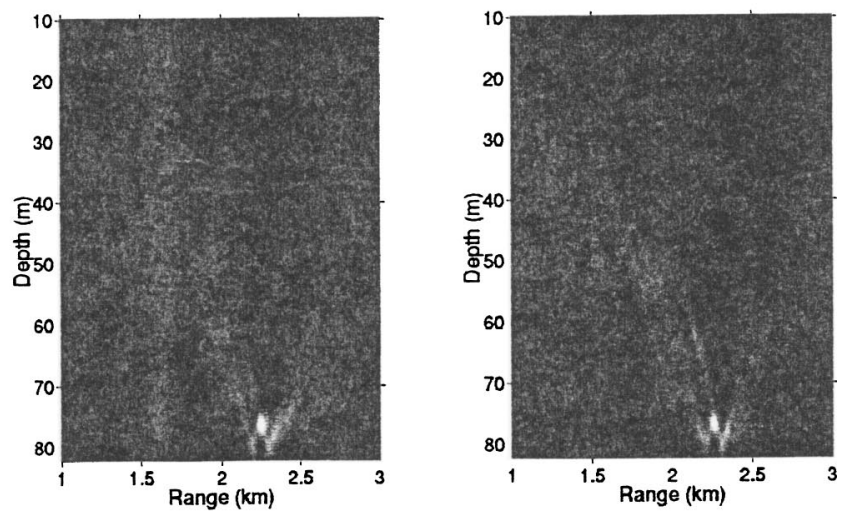

FIG. 3. Incoherent Bartlett ambiguity surfaces obtained for the $2 \mathrm{~km}$ track using six multitone frequency bins in the band 800 to $1500 \mathrm{~Hz}$. B is the maximum Bartlett power obtained in each ambiguity surface.

channel cannot be taken into account by inverting the seafloor. Therefore, for the remainder of this study seafloor properties are not included in the search space.

\section{The $5 \mathrm{~km}$ track}

Having obtained stable localization results for the $2 \mathrm{~km}$ data set, the next step is to study the effect of a larger sourcereceiver range using the same procedure. First, the low frequency multitone data was processed in step (1) for the array position. Next, ambiguity surfaces were computed using the 
TABLE II. Summary of the source localization results for the 2, 5, and 10 $\mathrm{km}$ tracks in terms of the mean and standard deviation. The data used are the higher frequency MT (800-1500 Hz) transmissions.

\begin{tabular}{|c|c|c|c|c|c|c|}
\hline \multirow[b]{2}{*}{ Parameter } & \multicolumn{2}{|c|}{$2 \mathrm{~km}$} & \multicolumn{2}{|c|}{$5 \mathrm{~km}$} & \multicolumn{2}{|c|}{$10 \mathrm{~km}$} \\
\hline & mean & std & mean & std & mean & std \\
\hline Source range $(\mathrm{km})$ & 2.23 & 0.041 & 5.44 & 0.048 & 10.6 & 0.24 \\
\hline Source depth (m) & 76.5 & 0.22 & 75.6 & 0.37 & 74.1 & 1.6 \\
\hline Bartlett power & 0.57 & 0.072 & 0.60 & 0.047 & 0.37 & 0.057 \\
\hline
\end{tabular}

optimized parameters and these are shown in Fig. 5. The figure shows a well resolved peak that correctly localizes the source in both range and depth.

Ambiguity surfaces were then computed for the higher frequency MT data set [after the step-(1) otimization of the geometry] and these are shown in Fig. 6. In this case the algorithm completely failed to localize the source. Close observation of the surfaces reveals that in the first half of the run there is an ambiguity structure (peaks and sidelobes of maximum correlation) close to the expected structure from the low frequency results (see Fig. 5), and also that surfaces (4) and (5) have the peak at the correct location. The structure that appears in surface (1) to (6) disappears in surfaces (7) to (12) and this causes the true source location to be completely missed. This result suggests mismatch in environmental model used in the second half of the run.

To help mitigate the environmental mismatch problem, the water column sound speed profile was also parametrized.
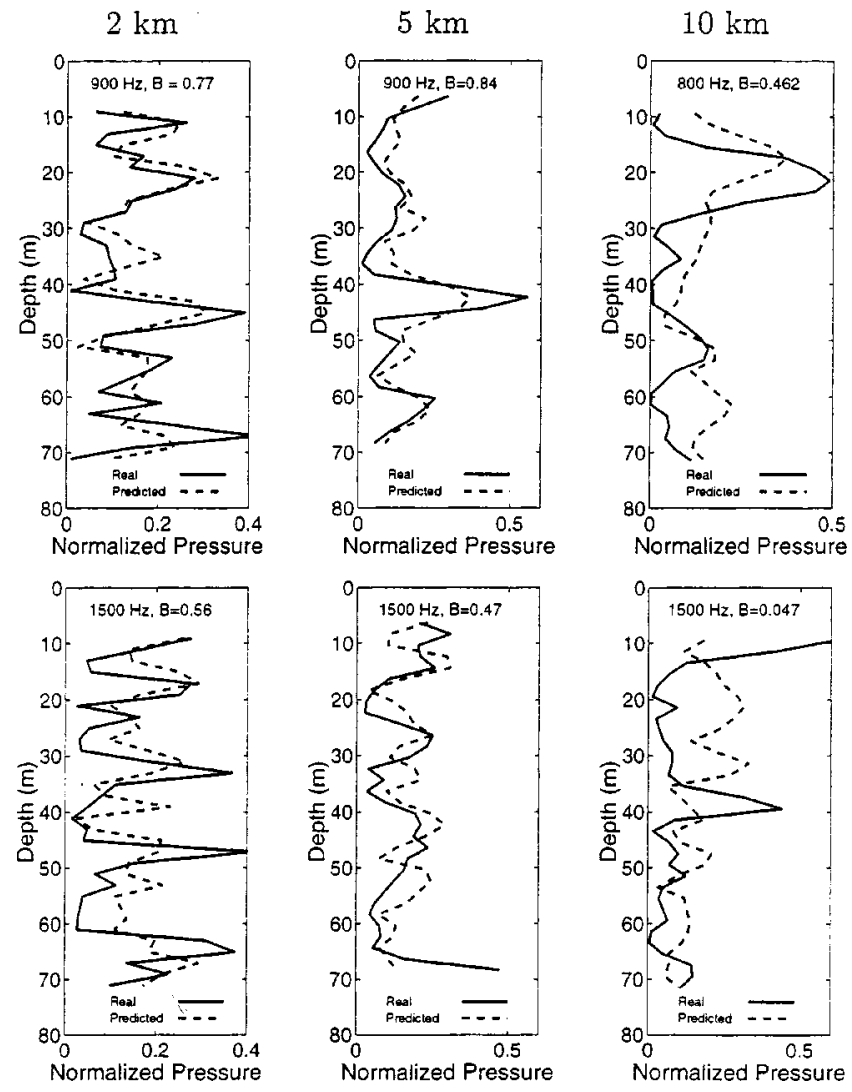

FIG. 4. Comparison of the predicted pressure with the measured pressure at frequencies with best and worst match for each range. The single frequency Bartlett power is marked on each plot.
$(1, \mathrm{~B}=0.76)$

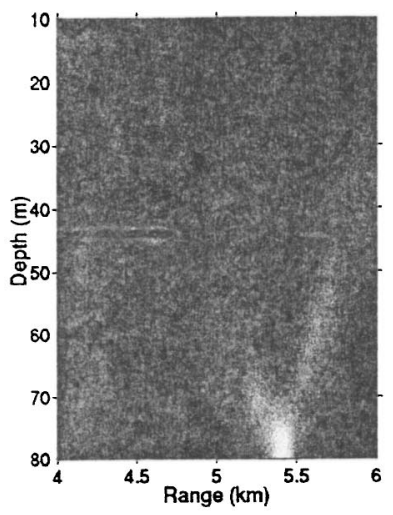

$(5, \mathrm{~B}=0.64)$

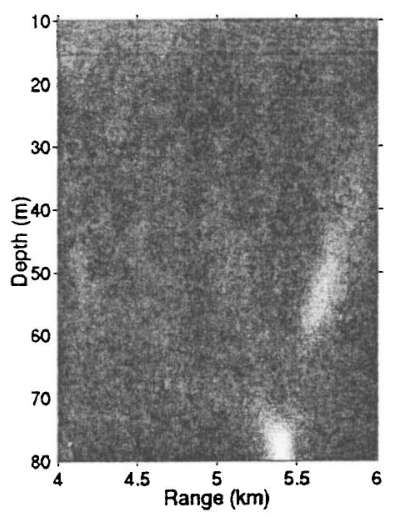

$(9, \mathrm{~B}=0.80)$

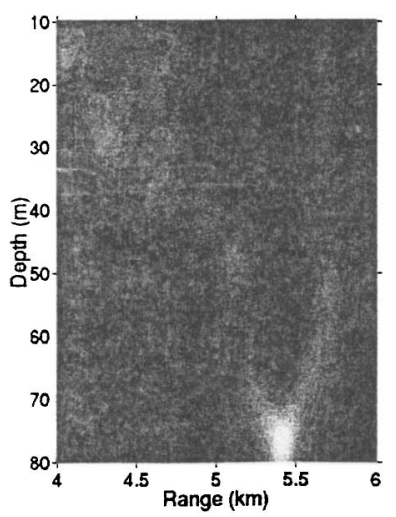

$(3, \mathrm{~B}=0.63)$

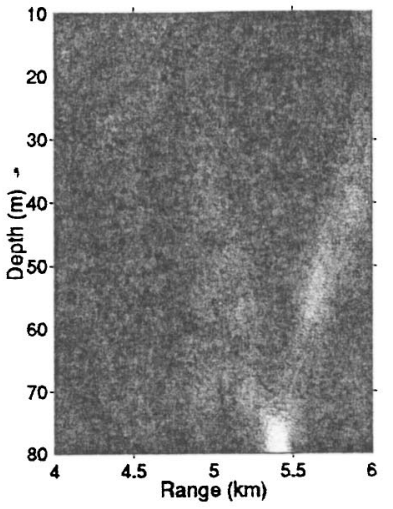

$(7, \mathrm{~B}=0.73)$

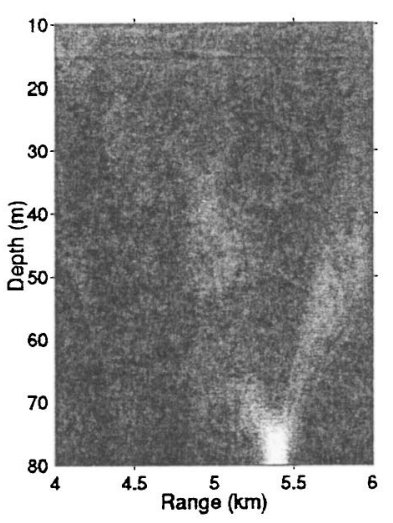

$(11, \mathrm{~B}=0.72)$

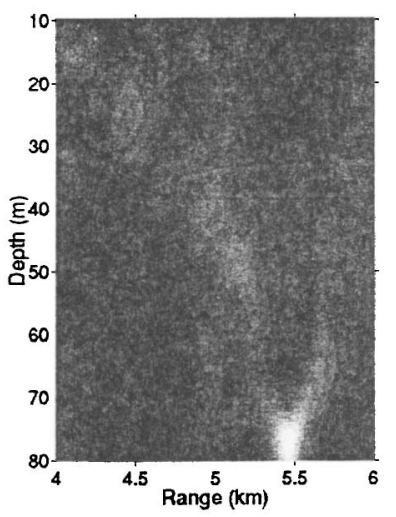

FIG. 5. Incoherent Bartlett ambiguity surfaces obtained for the $5 \mathrm{~km}$ track using six multitone frequency bins in the band 200 to $700 \mathrm{~Hz}$. B is the maximum Bartlett power obtained on each ambiguity surface.

This increases the degrees of freedom of the environmental model and allows the step-(1) search to find an effective sound speed and adjust to the time varying acoustic receptions. The sound speed profile can be parametrized in several ways and combined with site measurements. To correct for possible depth errors (and short-term fluctuations) in the towed CTD chain, a search over $4 \mathrm{~m}$ was included in the depth of the sound speed profile measurements used in the model. Another parameter included in the search space was the gradient of the sound speed profile in the portion between $67 \mathrm{~m}$ and the bottom, which is a region of the water column not covered by actual measurements. This is possibly very 
$(1, \mathrm{~B}=0.31)$

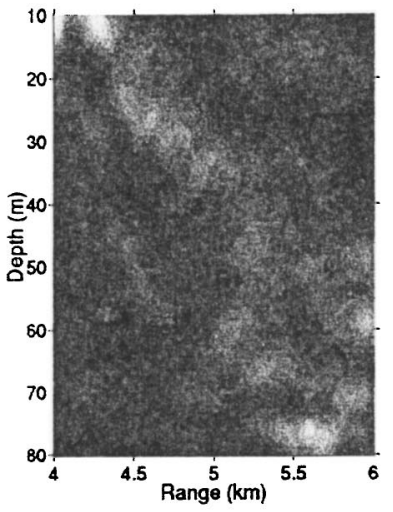

$(5, \mathrm{~B}=0.32)$

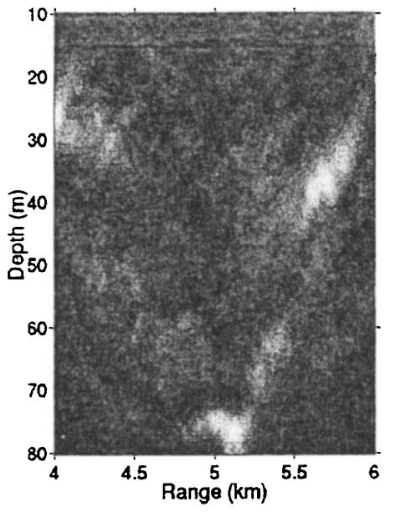

$(9, \mathrm{~B}=0.37)$

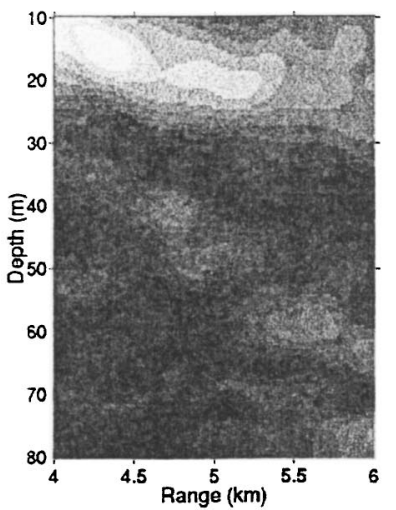

$(3, \mathrm{~B}=0.26)$

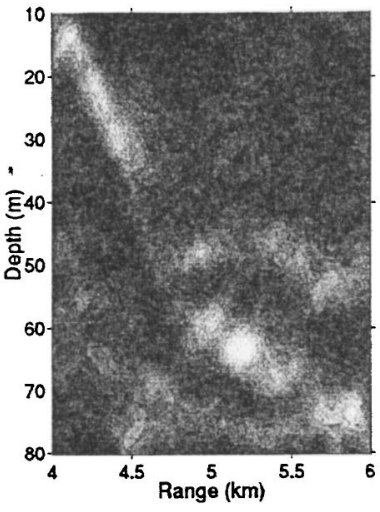

$(7, \mathrm{~B}=0.30)$

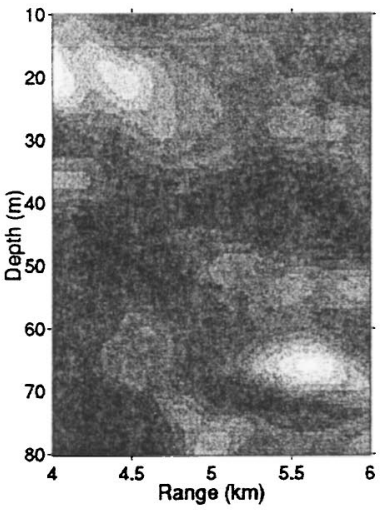

$(11, \mathrm{~B}=0.30)$

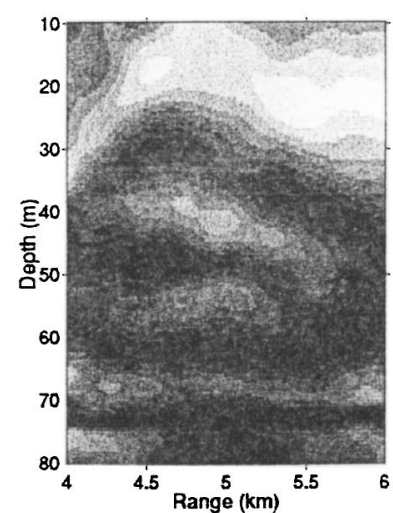

FIG. 6. Incoherent Bartlett ambiguity surfaces obtained for the $5 \mathrm{~km}$ track using six multitone frequency bins in the band 800 to $1500 \mathrm{~Hz}$. B is the maximum Bartlett power obtained on each ambiguity surface.

important for predicting the acoustic field since the sound source was located near the bottom. For the $2 \mathrm{~km}$ track, the sound speed profile was completed by extrapolating the two deepest sound speed values down to the bottom. Including the gradient as a search parameter implicitly assumes that the sound speed behaves linearly as the depth increases but should provide a better description. Even if this is a simple and crude assumption, this parametrization was judged sufficient to model the last values of the sound speed close to the bottom. The third and last attempt to track the time variability of the acoustic data in the $5 \mathrm{~km}$ high frequency data
$(1, \mathrm{~B}=0.60)$

$(3, \mathrm{~B}=0.63)$

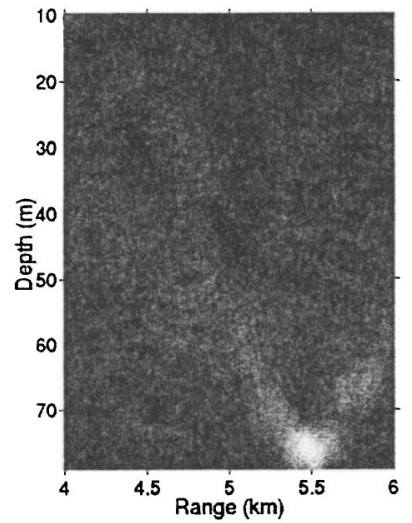

$(5, \mathrm{~B}=0.60)$

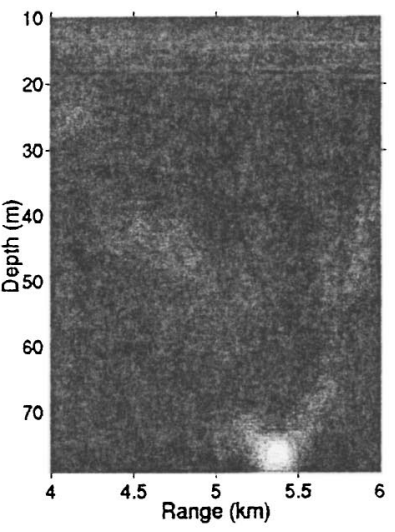

$(9, \mathrm{~B}=0.60)$

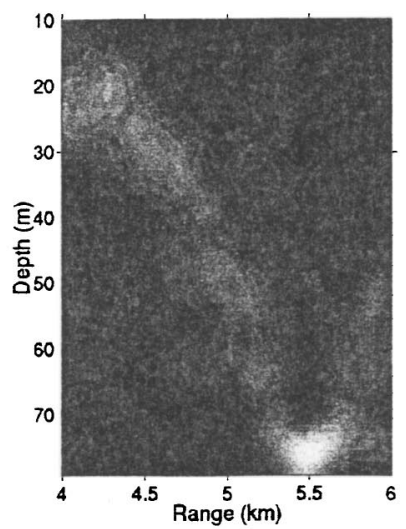

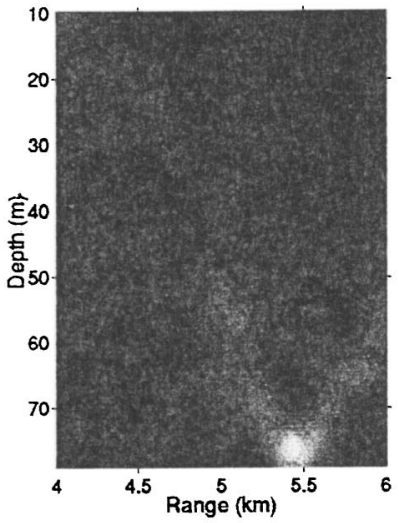

$(7, \mathrm{~B}=0.51)$

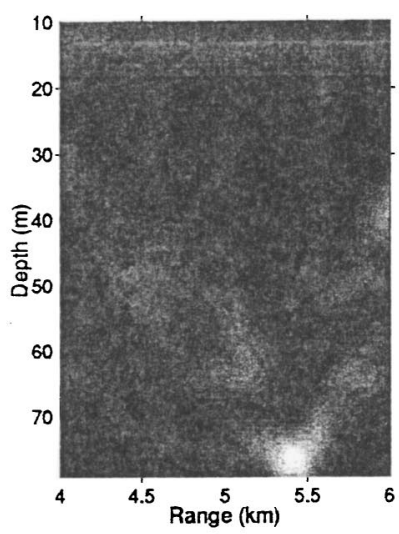

$(11, \mathrm{~B}=0.55)$

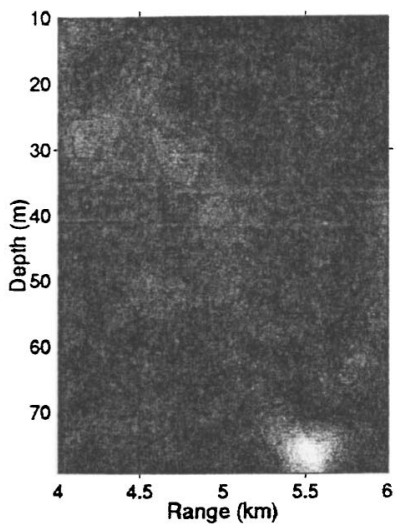

FIG. 7. Incoherent Bartlett ambiguity surfaces obtained for the $5 \mathrm{~km}$ track using six multitone frequency bins in the band 800 to $1500 \mathrm{~Hz}$. The focalization step included estimation of the sound speed profiles via EOFs. $B$ is the maximum Bartlett power obtained on each ambiguity surface.

set was to include a full parametrization of the sound speed profile evolution through time using a set of data based empirical orthogonal functions (EOFs). EOFs are basis functions ${ }^{9}$ that can be obtained from a database and are very efficient to reduce the number of data points to be estimated. If historical data is available, an efficient parametrization in terms of EOFs should lead to a faster convergence and better resolution in the optimal solution since a large amount of information is already available and the search is started 
(1)

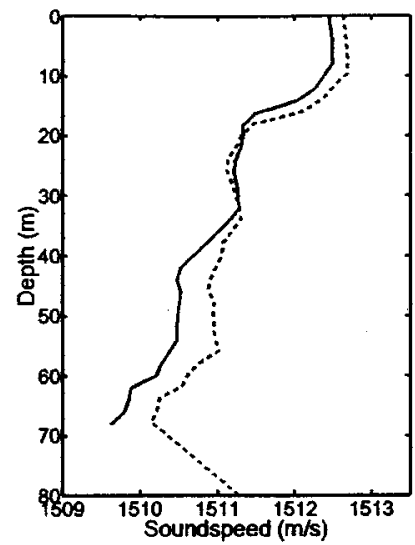

(5)

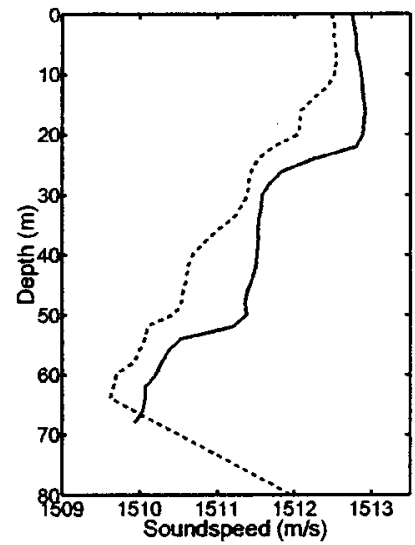

(9)

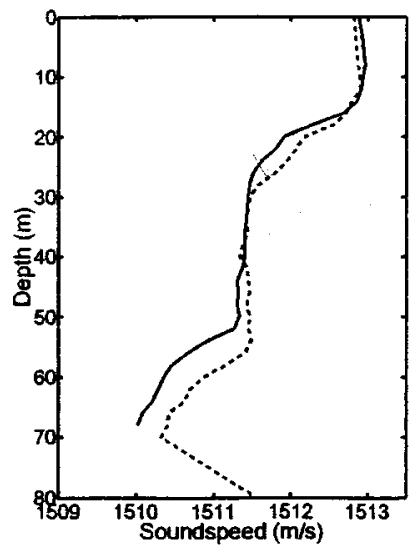

(3)

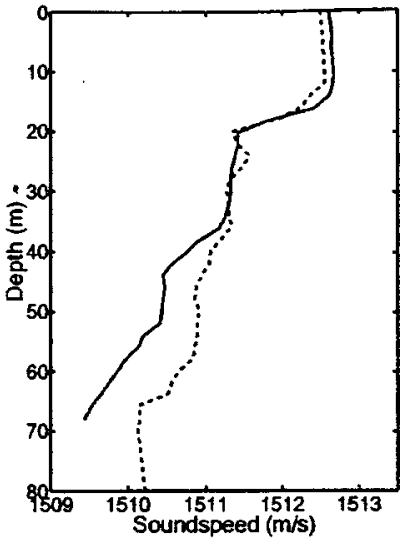

(7)

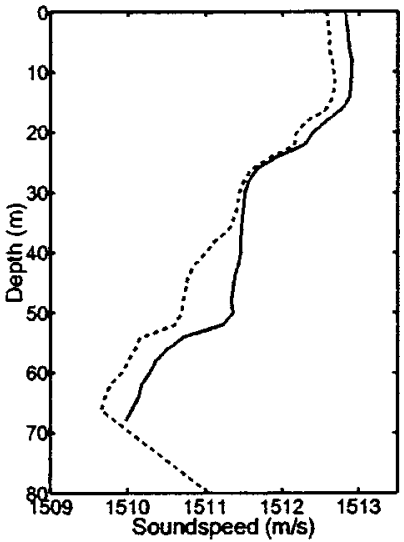

(11)

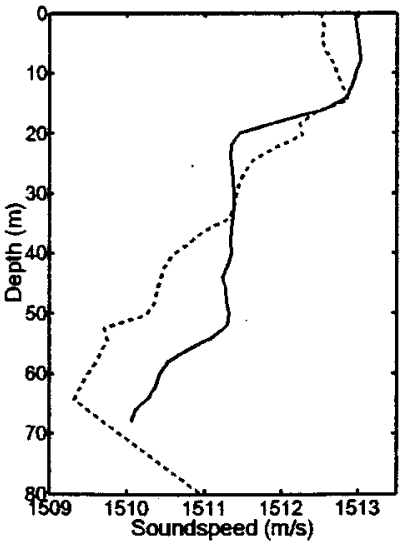

FIG. 8. Comparing sound speeds obtained by interpolation of real sound speeds with sound speeds estimated through EOFs using the higher frequency MT $(800$ to $1500 \mathrm{~Hz}$ ) at the $5 \mathrm{~km}$ track.

closer to the solution. The EOFs are constructed from representative data by sampling the depth dependence of the ocean sound speed. To account for the variability of the sound speed, measurements taken over time and at different locations are considered. The EOFs can be obtained from a singular value decomposition of the sample covariance matrix $\mathbf{C}$ as

$$
\mathbf{C}=\mathbf{U} \mathbf{\Lambda} \mathbf{U}^{\mathbf{H}} \text {, }
$$

where $\boldsymbol{\Lambda}=\operatorname{diag}\left[\lambda_{1}, \ldots, \lambda_{N}\right]$ is a diagonal matrix with the eigenvalues $\lambda_{n}, \mathbf{U}=\left[\underline{U}_{1}, \ldots, \underline{U}_{N}\right]$ is a matrix with orthogonal

$(1, \mathrm{~B}=0.65)$

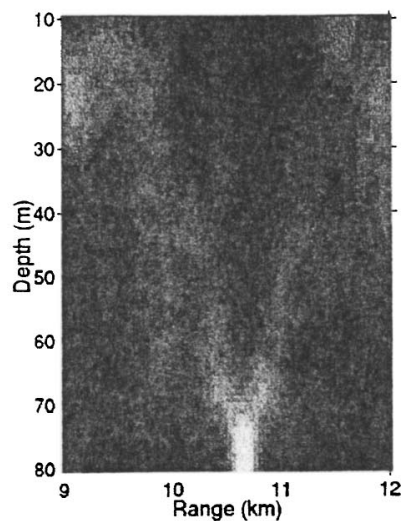

$(5, \mathrm{~B}=0.60)$

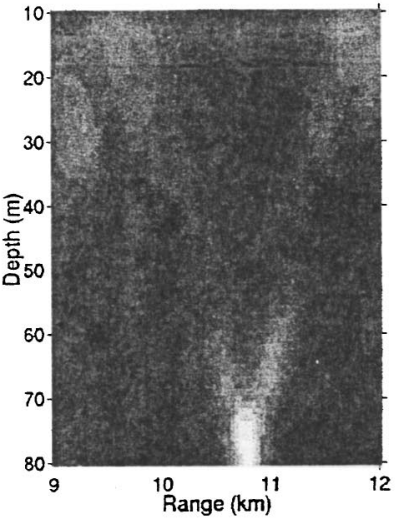

$(9, \mathrm{~B}=0.65)$

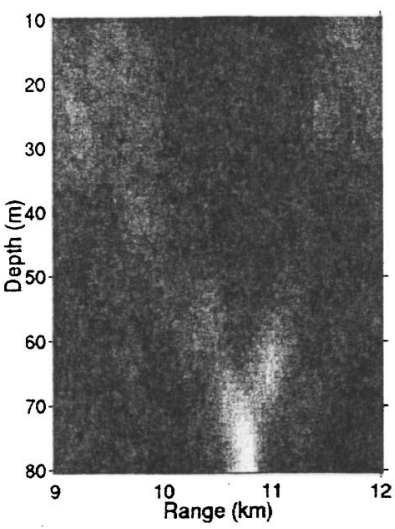

$(3, \mathrm{~B}=0.60)$

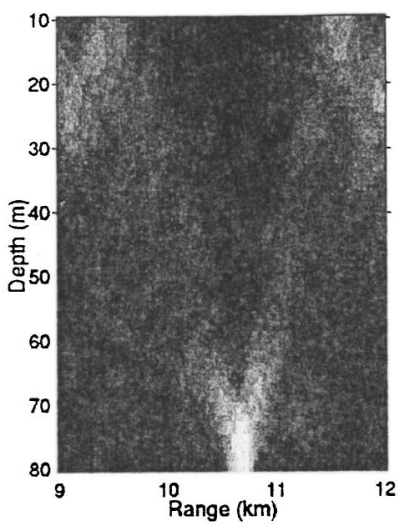

$(7, \mathrm{~B}=0.65)$

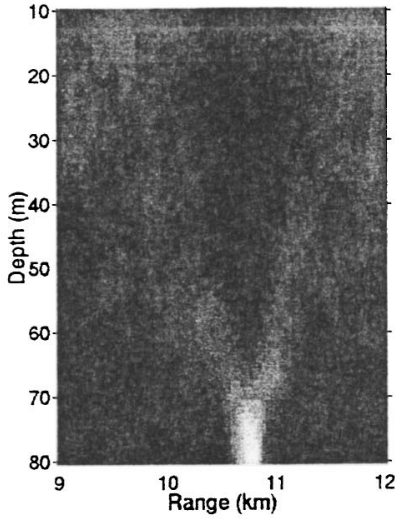

$(11, \mathrm{~B}=0.70)$

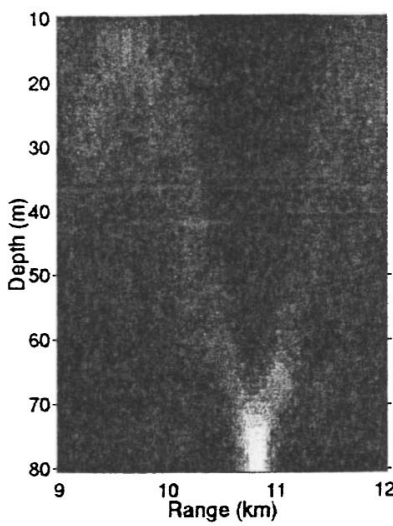

FIG. 9. Incoherent Bartlett ambiguity surfaces obtained for the $10 \mathrm{~km}$ track using six multitone frequency bins in the band 200 to $700 \mathrm{~Hz}$. The focalization step included estimation of the sound speed profiles via EOFs. $B$ is the maximum Bartlett power obtained on each ambiguity surface.

columns, which are used as the EOFs, $\mathbf{H}$ denotes conjugate transpose, and $N$ is the number of data points in the water column. Using the sound speed measurements taken close to the vertical array, it was found that three EOF's accounted for more than $80 \%$ of the water column energy. The modeled sound speed is written as

$$
\underline{C}_{\mathrm{EOF}}=\underline{\bar{c}}+\sum_{n=1}^{3} \lambda_{n} \underline{U}_{n}
$$


$(1, \mathrm{~B}=0.43)$

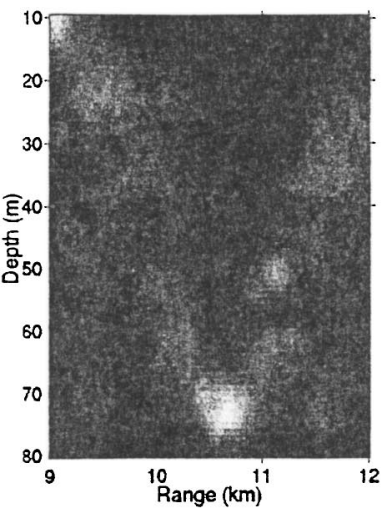

$(5, \mathrm{~B}=0.33)$

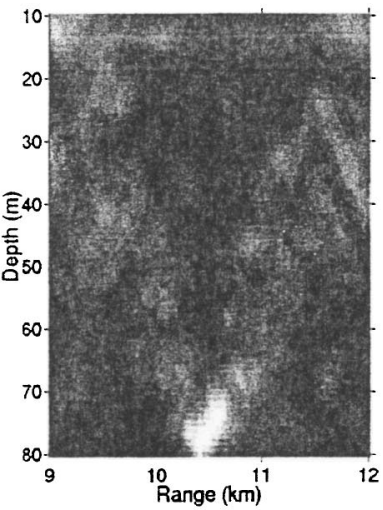

$(9, \mathrm{~B}=0.36)$

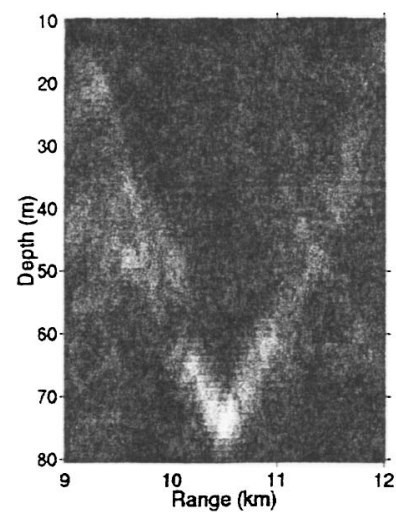

FIG. 10. Incoherent Bartlett ambiguity surfaces obtained for the $10 \mathrm{~km}$ track using MT frequency bins in the band 800 to $1500 \mathrm{~Hz}$. The focalization step included estimation of the sound speed profiles via EOFs. $B$ is the maximum Bartlett power obtained on each ambiguity surface.

where $\bar{c}$ is the average sound speed profile. By trial and error, the search interval for the coefficients $\lambda_{n}$ combining the (previously normalized) EOFs was chosen between -5 and 5 .

The total number of parameters included in the focalization step is now nine, four concerning the geometry, and five concerning the sound speed in the water column, corresponding to a search space with size $2 \times 10^{15}$. Regarding the GA optimization and in order to cope with this larger search space, the number of iterations was set to 40 , the number of individuals was set to 140, and the number of independent
$(1, \mathrm{~B}=0.39)$

$(3, \mathrm{~B}=0.43)$

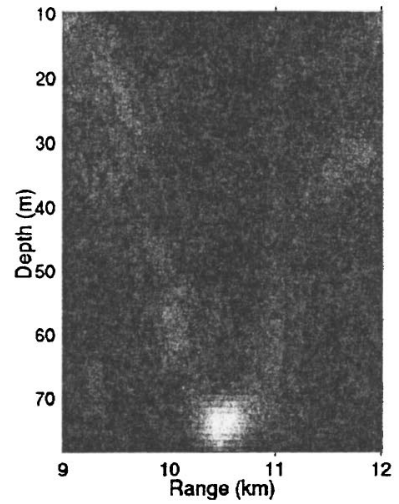

$(5, \mathrm{~B}=0.31)$

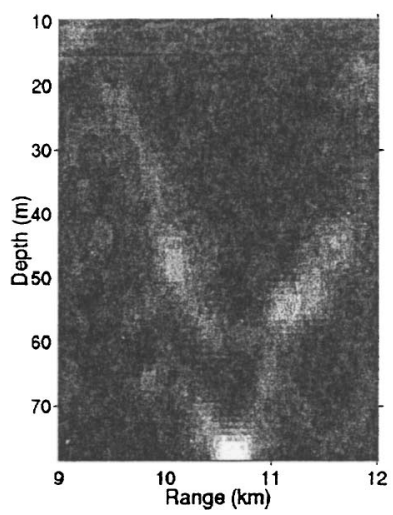

$(9, \mathrm{~B}=0.26)$

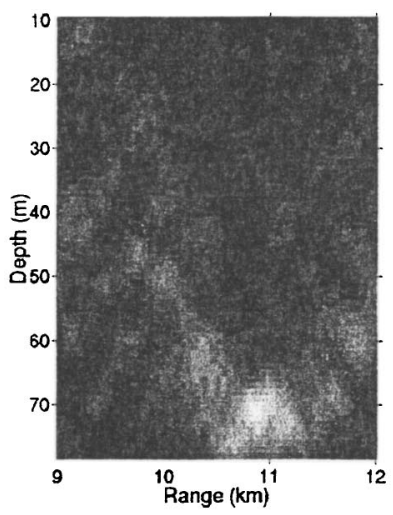

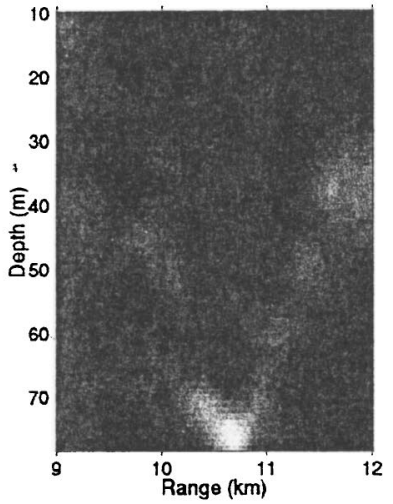

$(7, \mathrm{~B}=0.29)$

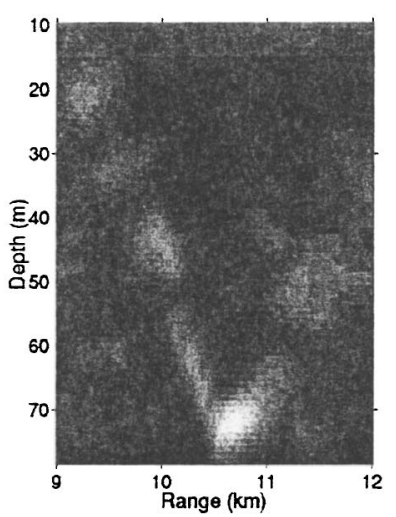

$(11, \mathrm{~B}=0.34)$

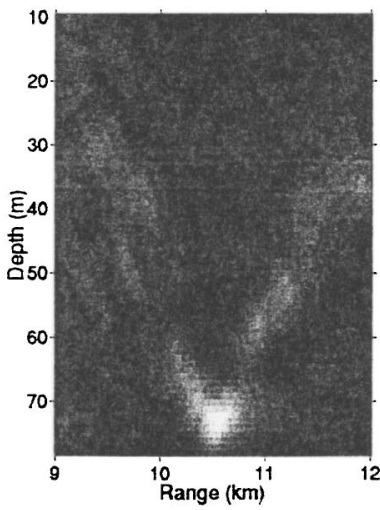

FIG. 11. Ambiguity surfaces obtained with the broadband conventional incoherent processor for the $10 \mathrm{~km}$ track using the higher frequency LFM signals ( 820 to $1500 \mathrm{~Hz}$ ), spaced by $23.4 \mathrm{~Hz}$. $B$ is the maximum Bartlett power obtained on each ambiguity surface.

populations was three which corresponds to about $1.5 \times 10^{4}$ forward models to be computed.

Observing the ambiguity surfaces obtained after accomplishing the focalization step (Fig. 7), it can be seen that the main peak is always nearly at the correct location, within negligible variations, and the ambiguities are largely suppressed. In Table II it can be seen that the variability of the source parameters is of the same order of greatness as those obtained for the $2 \mathrm{~km}$ track and that the mean Bartlett power is even higher than that obtained for the $2 \mathrm{~km}$ track. Figure 8 shows plots comparing the sound speed profiles measured 
(a)

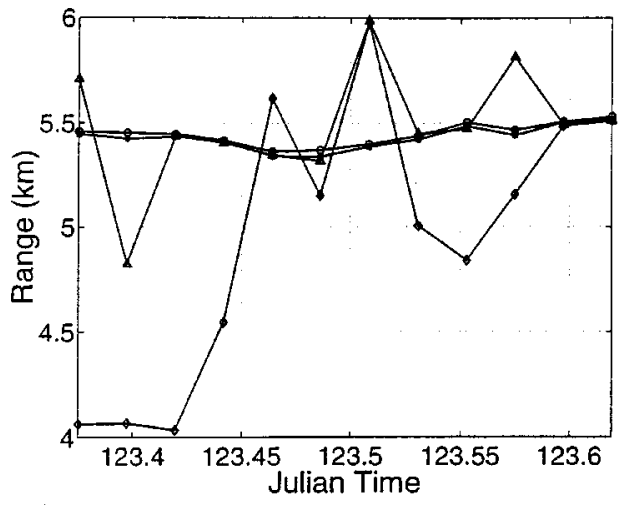

(b)

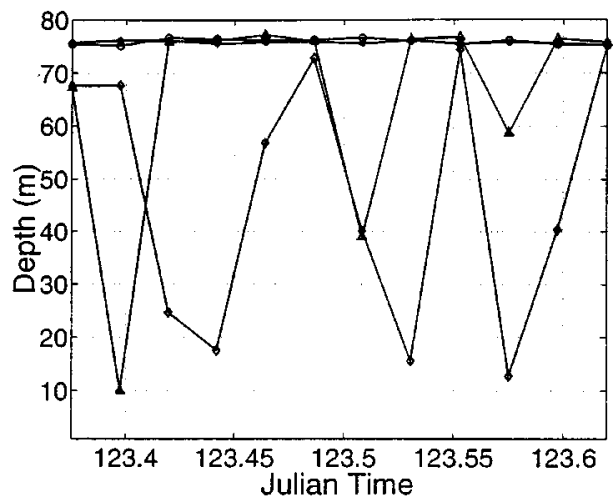

FIG. 12. Source localization results over time for the $5 \mathrm{~km}$ track for different array apertures using the higher frequency MT (800-1500 Hz): (a) Range; (b) depth. Each color stands for a different number of sensors: 31 $(\bigcirc) ; 16(*) ; 8(\triangle) ; 4(\diamond)$ close to the array and those obtained using the EOF expansion. In general it can be seen that there is a significant difference between the sound speed profiles being compared. There are at least two reasons to explain this difference: one is that the estimated profiles result from an integral of the sound speed profiles along the acoustic propagation path, and therefore include water column range dependency, if any, the other is that the estimated profiles result from a multiparameter optimization procedure and therefore may accommodate possible deviations of other environmental and/or geometric parameters, yielding the best matched-field response. An example is the sound speed profile gradient below $67 \mathrm{~m}$ (Fig. 8), that has often a nonphysical steep increase towards the bottom. In this paper there is no intention in validating the optimized sound speed profiles.

Finally, Fig. 4 shows that for this track the replica field at $900 \mathrm{~Hz}$ is well matched with the received acoustic field, and that the match at $1500 \mathrm{~Hz}$ is reasonable. The parametrization chosen for the sound speed allowed therefore for modeling the environment such that localization results of high quality could be obtained for the high frequency data set.

\section{The $10 \mathrm{~km}$ track}

It was shown that the modeling scheme applied at $2 \mathrm{~km}$ failed to accurately localize the source at $5 \mathrm{~km}$ for the higher frequency data set. The strategy for environmental focalization followed for the $5 \mathrm{~km}$ track has shown to be effective for that track, i.e., the replica fields were successfully matched with the field received by the array. Now the question is whether the focusing method applied at $5 \mathrm{~km}$ works also at $10 \mathrm{~km}$.

The optimization strategy was therefore repeated for the $10 \mathrm{~km}$ track data where the only difference is the temperature data set from which the EOFs are computed. Previously, since the sound speed profiles used were only those measured close to the array, only time variability was taken into account. For this track to obtain the EOFs, a database of sound speed profiles was constructed with profiles updated every 5 minutes and comprising the whole $10 \mathrm{~km}$ track acquisition period. Note that, as the CTD chain was towed continuously between source and vertical array this database includes profiles that are both range and time dependent. As previously, it was found that the first three EOFs were sufficient to represent the temperature field.

The focalization step included the geometric parameters of Table I and the sound speed profile parameters as explained in Sec. II. The results obtained on the lower frequency data set are shown in Fig. 9 and indicate that the source location is well resolved over the whole time series, and the main peak is well above the background ambiguities. The source range was estimated with a mean value of 10.6 $\mathrm{km}$ with a small variation of approximately $\pm 50 \mathrm{~m}$, and a source depth estimate with a variation of about $3 \mathrm{~m}$ around the true value of $76 \mathrm{~m}$. The Bartlett power over time is larger than 0.6, which means that the agreement between the model and the measured data is relatively good.

Then, the same two step procedure was applied to the higher frequency band data set. In order to reduce the mismatch possibly due to the higher frequency and the longer range, the water depth was also included in the focalization step in agreement with the search interval shown in Table I. The ambiguity surfaces obtained for the MT analyzed are shown in Fig. 10. It can be noticed that the main peak is not completely stable in its correct position, but it always appears close to the correct location. The standard deviation is about $240 \mathrm{~m}$ in range and $1.6 \mathrm{~m}$ in depth (Table II). This corresponds to an increase relative to the $5 \mathrm{~km}$ track, as expected, since over a higher distance the environmental variabilities are more significant. As it can be seen in Fig. 4, there are significant difficulties, in particular at $1500 \mathrm{~Hz}$, where there is no match at all (Barlett power is 0.047).

The source localization experimental results obtained for the MT are satisfactory with, however, a considerable increase of range and depth uncertainty with increasing range and with the high frequency data set. The issue is to know whether the modeling has reached its limitations, i.e., one can question whether the degree of sophistication of a rangeindependent environmental model is high enough to model this waveguide. For example, the hypothesis of a partially range-dependent model could be considered. Another hypothetical issue is whether it is possible to increase the amount of information inserted in the localization process by using more frequencies. Experimental results have shown that increasing the number of frequencies results in more consistent source localization estimates..$^{9,10,16,17}$ In order to test that possibility, the LFM sweeps emitted in the same time slots as the 
MT were processed. A number of 30 frequencies ranging from 820 to $1500 \mathrm{~Hz}$ with a spacing of $23.4 \mathrm{~Hz}$ were selected. It can be seen that while for the tones a sustained variability in range is present, with the LFM signals it is possible to achieve periods of constancy (Fig. 11).

\section{The effect of array aperture}

After working with an array spanning around three quarters of the water column, and having obtained stable localization results at three different ranges, the goal now is to study the dependency of the MFP procedure on the array aperture. It is well known that, as the aperture is decreased less spatial discrimination is obtained, hence resulting in higher sidelobes relative to the main peak. In theory, even if the main peak-to-sidelobe ratio decreases with the array aperture, the main peak will always be at the correct position. However, in practice, with real data, geometric and environmental mismatch is always present and the acoustic field is observed during a limited time which creates a situation where a decrease of array aperture may indeed lead to a failure of source localization.

In the ADVENT'99 data set, reduced array aperture MFP was first applied to the higher frequency MT of the 5 $\mathrm{km}$ track, since for this range stable results with the full array were achieved, and could be used as reference. The array configuration used up to now had 31 elements spaced by 2 $\mathrm{m}$. The procedure adopted to decrease the array aperture is to leave out sensors from the top and the bottom in such a way that the array is always centered in the water column, keeping the same spacing between sensors. The environment and geometry used were those obtained in the last focalization step for this range, hence exhaustive search is being carried out only for range and depth.

Figure 12 shows the results obtained by reducing the array aperture by successive factors of 2, i.e., from the full 31 sensors to 16 , then 8 and finally to 4 sensors. The curve with circles shows the result obtained previously with the full aperture. Comparing the curves for range and depth obtained with half aperture (asterisks) to those obtained with full aperture, only negligible difference is noticed. For eight sensors (14 $\mathrm{m}$ of array aperture) it is still possible to obtain reasonable source localization results (curve with triangles), with, however, a larger spread in the estimates. The four

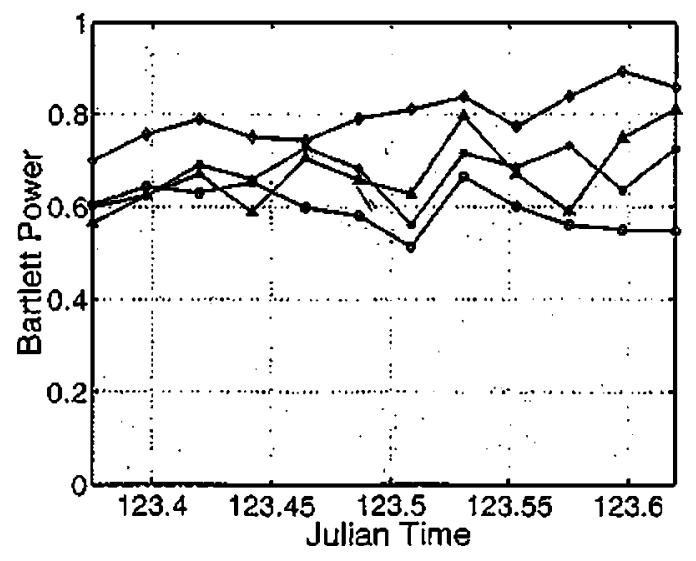

FIG. 13. Bartlett power over time for the $5 \mathrm{~km}$ track for different array apertures using the higher frequency MT (800-1500 Hz). Each color stands for a different number of sensors: $31(\bigcirc) ; 16(*) ; 8(\triangle) ; 4(\diamond)$.

sensors case (6 m aperture) represents a sampling of about $8 \%$ of the water column (curve with diamonds) where a significant degradation can be noticed.

Notice that the Bartlett power is highest for the four sensor configuration over the whole time even if the MFP procedure failed (Fig. 13). The increment of the Bartlett power with the reduction of the array aperture is due to the reduced complexity of the acoustic field as seen by the array. The matched-field response is a measure of the similarity of the real acoustic field with the replicas produced by the numerical propagation model fed with different source locations. If the aperture is small, then the uniqueness of the acoustic field along the portion of water column spanned by the field is lower, hence the match is higher.

Again, there is the possibility of compensating the lack of aperture by the addition of a large number of frequencies. Taking the LFM signals, a continuous set of frequencies is available, therefore 30 equally spaced frequencies between 820 and $1500 \mathrm{~Hz}$ were extracted and processed in the worst case situation of only four sensors. Figure 14 shows the improvement obtained. There are still three outliers and some variability, but now it is possible to guess that 5.4 or $5.5 \mathrm{~km}$ might be the correct value for range and that the source depth might be between 70 and $80 \mathrm{~m}$. This case shows that it was possible to partially compensate the lack of information obtained by reduced spatial sampling by increasing the number of frequencies and obtain a reasonable source localization at (a)

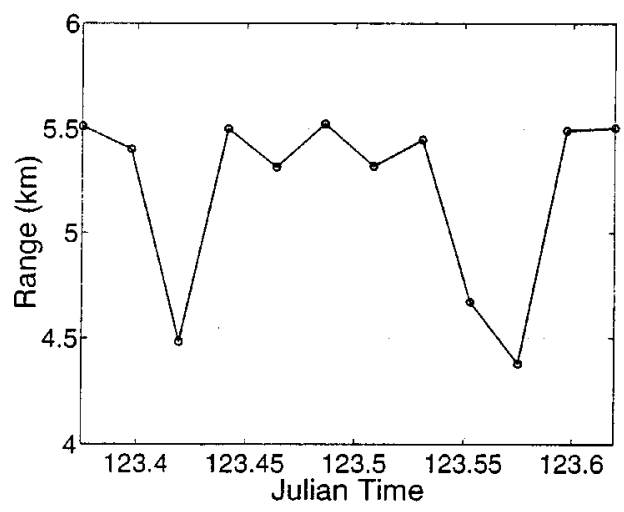

(b)

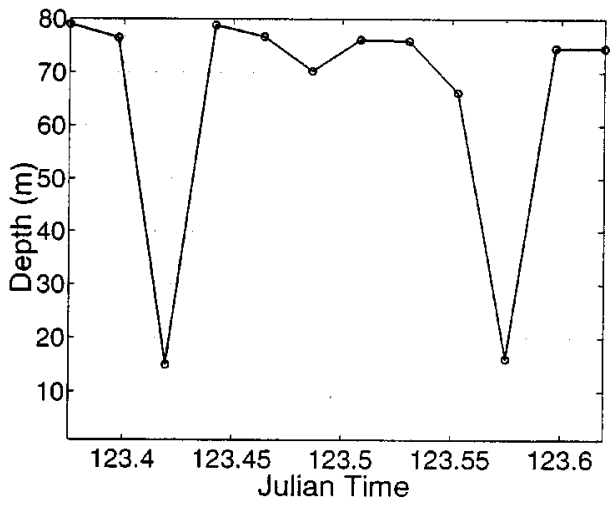

FIG. 14. Source localization results over time for an array aperture of $6 \mathrm{~m}$ using the higher frequency LFM signals (820-1500 Hz, spaced every 23.4 $\mathrm{Hz}$ ): (a) range; (b) depth. 
$5 \mathrm{~km}$ from a $6 \mathrm{~m}$ aperture vertical array with only four sensors ( $8 \%$ of the water column). Using the environmental parameter set obtained during the focalization step with 31 hydrophones strongly contributed for this result.

\section{CONCLUSION}

Acoustic data were collected in a $80 \mathrm{~m}$ depth mildly range-dependent shallow water area of the Strait of Sicily, during the ADVENT'99 sea trial in May 1999. A vertical line array was deployed at three different ranges of 2, 5, and 10 $\mathrm{km}$ from an acoustic source. A series of multitone and linear frequency modulated sweeps were transmitted in two frequency bands of 200 to $700 \mathrm{~Hz}$ and 800 to $1500 \mathrm{~Hz}$.

A two stage matched-field processing (MFP) algorithm was applied throughout: (1) Focalization using genetic algorithms to search for the array position and environmental parameters giving the best fit between measured data and modeled replicas. (2) Compute the MFP ambiguity surfaces in range and depth for source localization using previously determined array position and environmental characterization.

Concerning the analysis of the ADVENT'99 data, one of the conclusions in Ref. 11 is that the environmental variability at longer ranges can destroy coherent processing and propagation prediction of acoustic data. This paper completes Ref. 11 with the following conclusions: (1) As the source range and frequency are increased, the water column variability becomes more important and this needs to be accounted for in the modeling to obtain a good match between data and replica. (2) The water column variability can be modeled using an EOF expansion and estimated with the focalization process. (3) With a full sound speed focalization precise MFP localizations could be obtained at all ranges and in both frequency bands (frequencies up to $1.5 \mathrm{kHz}$ ). (4) The bottom parameters can be estimated from the short range 2 $\mathrm{km}$ track (where variability and range dependence is less problematic) and held constant for all the other sourcereceiver ranges up to $10 \mathrm{~km}$.

The attempt to decrease the array aperture showed that at $5 \mathrm{~km}$ source range, it was possible to achieve nearly correct localizations with an array 4 times smaller than the initial full aperture array, sampling only $\frac{1}{6}$ of the water column. Using an increased number of frequencies allowed reducing the array aperture even further using only four sensors $\left(\frac{1}{12}\right.$ of the water column).

The results reported in this paper indicate that under controlled conditions and in a shallow water environment of low range dependence, it is possible to accurately model the acoustic field for ranges up to $10 \mathrm{~km}$ and frequencies up to $1.5 \mathrm{kHz}$ to correctly localize an acoustic source over time if the optimized modeling process has taken into account possible time variabilities in the water column. Moreover, these results indicate that the MFP technique can be made robust to model mismatch caused by the water column time dependence, if an adequate focalization procedure is incorporated, even at high frequencies and ranges up to $10 \mathrm{~km}$. This indicates that it may be feasible to carry out source localization at frequencies higher than typically considered for MFP even in the presence of strong environmental fluctuations such as internal tides, eddies, and fronts, if sufficient a priori information on the water column is available.

\section{ACKNOWLEDGMENTS}

The authors would like to thank SACLANTCEN for the support given during this study, in particular to Jürgen Sellschopp (the chief scientist of the ADVENT'99 cruise), Peter Nielsen and the NRV Alliance crew. This work was partially supported by PRAXIS XXI, FCT, Portugal, under the ATOMS project, Contract No. PDCTM/P/MAR/15296/1999.

${ }^{1}$ H. P. Bucker, "Use of calculated sound fields and matched-field detection to locate sound source in shallow water," J. Acoust. Soc. Am. 59, 368373 (1976).

${ }^{2}$ M. Hinich, "Maximum likelihood signal processing for a vertical array," J. Acoust. Soc. Am. 54, 499-503 (1973).

${ }^{3}$ A. B. Baggeroer, W. A. Kuperman, and P. N. Mikhalevsky, "An overview of matched field methods in ocean acoustics," IEEE J. Ocean. Eng. 18, 401-424 (1993).

${ }^{4}$ R. M. Hamson and R. M. Heitmeyer, "An analytical study of the effects of environmental and system parameters on source localization in shallow water by matched-field processing of a vertical array," J. Acoust. Soc. Am. 86, 1950-1959 (1989).

${ }^{5}$ S. Jesus, "Normal-mode matching localization in shallow water: environmental and system effects," J. Acoust. Soc. Am. 90, 2034-2041 (1991).

${ }^{6}$ A. M. Richardson and L. W. Nolte, "A posteriori probability source localization in an uncertain sound speed deep ocean environment," J. Acoust. Soc. Am. 89, 2280-2284 (1991).

${ }^{7}$ M. D. Collins and W. A. Kuperman, "Focalization: Environmental focusing and source localization,” J. Acoust. Soc. Am. 90, 1410-1422 (1991).

${ }^{8}$ D. F. Gingras and P. Gerstoft, "Inversion for geometric parameters in shallow water: Experimental results," J. Acoust. Soc. Am. 97, 3589-3598 (1995).

${ }^{9}$ P. Gerstoft and D. F. Gingras, "Parameter estimation using multifrequency range-dependent acoustic data in shallow water," J. Acoust. Soc. Am. 99, 2839-2850 (1996).

${ }^{10}$ C. Soares, A. Waldhorst, and S. M. Jesus, "Matched-field processing: environmental focusing and source tracking with application to the North Elba data set," Oceans'99 MTS/IEEE Proceedings, Seattle, 1999, Vol. 3, pp. 1598-1602.

${ }^{11}$ M. Siderius, P. Nielsen, J. Sellschopp, M. Snellen, and D. Simons, "Experimental study of geo-acoustic inversion uncertainty due to ocean sound-speed fluctuations," J. Acoust. Soc. Am. 110, 769-781 (2001).

${ }^{12}$ T. Fassbender, "Erweiterte Genetische Algorithmen zur globalen Optimierung multi-modaler Funktionen," Ruhr-Universität, Master thesis, Bochum, Germany, 1995.

${ }^{13}$ J. Sellschopp, M. Siderius, and P. Nielsen, “ADVENT'99, pre-processed acoustic and environmental cruise data," SACLANTCEN Undersea Research Center, Internal Report CD-35, La Spezia, Italy, 2000.

${ }^{14}$ M. Snellen, D. G. Simons, M. Siderius, J. Sellschopp, and P. L. Nielsen, "An evaluation of the accuracy of shallow water matched field inversion results," J. Acoust. Soc. Am. 109, 514-527 (2001).

${ }^{15}$ C. M. Ferla, M. B. Porter, and F. B. Jensen, "C-SNAP: Coupled SACLANTCEN normal mode propagation loss model," SACLANTCEN Undersea Research Center, Memorandum SM-274, La Spezia, Italy, 1993.

${ }^{16}$ A. B. Baggeroer, W. A. Kuperman, and H. Schmidt, "Matched field processing: Source localization in correlated noise as an optimum parameter estimation problem," J. Acoust. Soc. Am. 83, 571-587 (1988).

${ }^{17} \mathrm{~S}$. Jesus, "Broadband matched-field processing of transient signals in shallow water," J. Acoust. Soc. Am. 93, 1841-1850 (1993). 